\title{
Evaluation of Tanshinone IIA Developmental Toxicity in Zebrafish Embryos
}

\author{
Tao Wang ${ }^{1}$, Chengxi Wang ${ }^{2}$, Qiong $\mathrm{Wu}^{2}$, Kangdi Zheng ${ }^{2}$, Jiaojiao Chen ${ }^{3}$, Yutao Lan ${ }^{3, *}$, \\ Yao Qin ${ }^{4}$, Wenjie Mei ${ }^{2, *}$ and Baoguo Wang ${ }^{1, *}$ \\ 1 School of Public Health, Guangdong Pharmaceutical University, Guangzhou 510310, Guangdong, China; \\ wangtao1234@126.com \\ 2 School of Pharmacy, Guangdong Pharmaceutical University, Guangzhou 510006, Guangdong, China; \\ gdpuwcx@163.com (C.W.); 13533260720@163.com (Q.W.); kangdizheng@163.com (K.Z.) \\ 3 School of Nursing, Guangdong Pharmaceutical University, Guangzhou 510310, Guangdong, China; \\ ch381286034@126.com \\ 4 Chn-Alternative Biotech Co. Ltd., Guangzhou 510432, Guangdong, China; chnalt@163.com \\ * Correspondence: lanyutao@gdpu.edu.cn (Y.L.); wenjiemei@126.com (W.M.); \\ gdwangbaoguo@163.com (B.W.); Tel.: +86-20-3407-4397 (Y.L.); +86-20-3935-2128 (W.M.); \\ +86-760-8820-7997 (B.W.)
}

Academic Editor: Julio A. Seijas Vázquez

Received: 25 February 2017; Accepted: 15 April 2017; Published: 21 April 2017

\begin{abstract}
Tanshinone IIA (Tan-IIA) is derived from the dried roots of Salvia miltiorrhiza Bunge, a traditional Chinese medicine. Although Salvia miltiorrhiza has been applied for many years, the toxicity of the mono-constituent of Salvia miltiorrhiza, tanshinone IIA, is still understudied. This study evaluated the cardiotoxicity and developmental malformations of Tan-IIA by using zebrafish normal embryos and dechorionated embryos. After treatment with Tan-IIA in different concentrations for four-day periods, obvious pericardial edema, spinal curvature, and even missing tails were observed in zebrafish embryos. The $\mathrm{LC}_{50}$ values in the dechorionated embryo group at $72 \mathrm{~h}$ post-fertilization (hpf) and $96 \mathrm{hpf}$ were $18.5 \mu \mathrm{M}$ and $12.8 \mu \mathrm{M}$, respectively, and the teratogenicity was manifested at a concentration of about $1 \mu \mathrm{M}$. The main endpoints of teratogenicity were scoliosis, malformation of tail, and pericardium edema. Our findings displayed the potential cardiotoxicity and severe impact on the abnormal development of Tan-IIA in zebrafish embryo at high concentrations, which may help avoid the risk of its clinical application.
\end{abstract}

Keywords: zebrafish; tanshinone IIA; developmental toxicity

\section{Introduction}

Tanshinone IIA (Tan-IIA), a fat-soluble fuchsia needle crystal, is the main active ingredient of diterpene quinone in traditional Chinese medicine Salvia miltiorrhiza. Because of its unique quinoid structure, Tan-IIA may be involved in multiple biochemical reactions of the organism and have a variety of biological activities. Therefore, Tan-IIA is widely used in the treatment of cardiovascular disease [1-3]. A large amount of researches show that Tan-IIA exhibits various pharmacological activities, including anti-inflammatory [4], anti-oxidative [5], anti-fibrosis [6], modulation of collagen metabolism [7], anti-tumor [8] and so on.

Zebrafish (Danio rerio) is a small tropical fish with the model advantages of large spawning, rapid breeding, and being easy to raise, etc. [9]. It has been recommended as an animal model for some standard toxicology tests. Evaluation of drug toxicity using zebrafish has the advantages of easy observation, low cost, simple operation, good repeatability, high sensitivity, and short experiment periods, and is an internationally recognized standard method of evaluation of drug 
toxicity [10]. Zebrafish embryo developmental toxicity is based on teratogenesis and mortality of the zebrafish embryos [11,12]. The current acute toxicity experiments are mainly concentrated in mice as a representative of the animal model. However, due to its high breeding conditions, high cost, complex operation, and ethical limits, it is difficult to achieve comprehensive urgent toxicity tests with this model $[13,14]$. Therefore, using the zebrafish model to evaluate drug toxicity is of great significance, and the zebrafish model has been widely used in the assessment of acute toxicity and developmental toxicity [15-20].

In a previous study, our group explored the acute toxicity of dimethyl sulfoxide (DMSO) by using the zebrafish embryo model [21], which could thus be utilized for choosing the safe concentration range of DMSO as a solvent [22]. This current study firstly investigated the developmental toxicity effect of Tan-IIA on the zebrafish embryo model. The proposed zebrafish acute toxicity assay is very valid and reliable for rapid evaluation of Tan-IIA toxicity, and saves time and cost during drug research and development.

\section{Results}

\subsection{The Crystal Structure of Tan-IIA}

Single crystal X-ray diffraction analysis revealed that Tan-IIA crystallized in the Pmna space group, and the data is summarized Table S1. The fundamental asymmetric group contained only one Tan-IIA molecule. As shown in Figure 1a, two dibenzopyrrole units displayed a large aromatic planar structure with one furan ring (ring a) and two benzene ring (ring b and c), which the hexamethylene group showed a classic chair conformation. Stacking interactions in a step showed strong overlap for Tan-IIA. As shown in Figure 1b, it is showed great inter-molecular interaction between two benzene rings with a diketone group (ring $b$ ) of two molecules occurring with distances to the ring centroid of $3.396 \AA$ by $\pi-\pi$ stacking interactions. This created an infinite 1D ribbon composed of Tan-IIA secondary building units (SBUs) running along the $b$ axis, as seen in Figure 1d. The layers consisted of a benzene ring with a diketone group positioned adjacent to each other, separated by $3.396 \AA$ via their $\pi-\pi$ stacking interactions, as seen in Figure 1d,e.

(a)

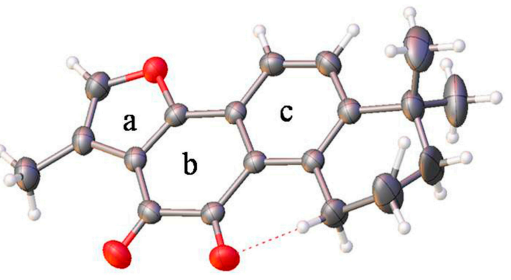

(c)

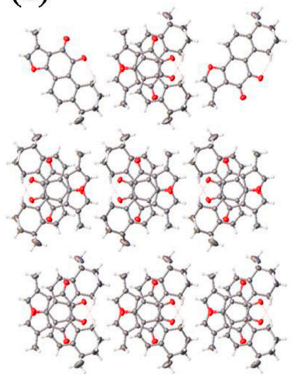

(d)

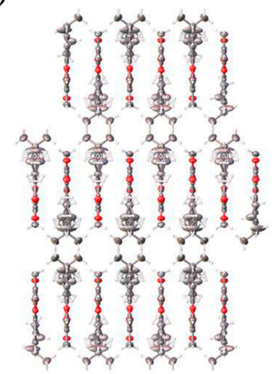

(b)
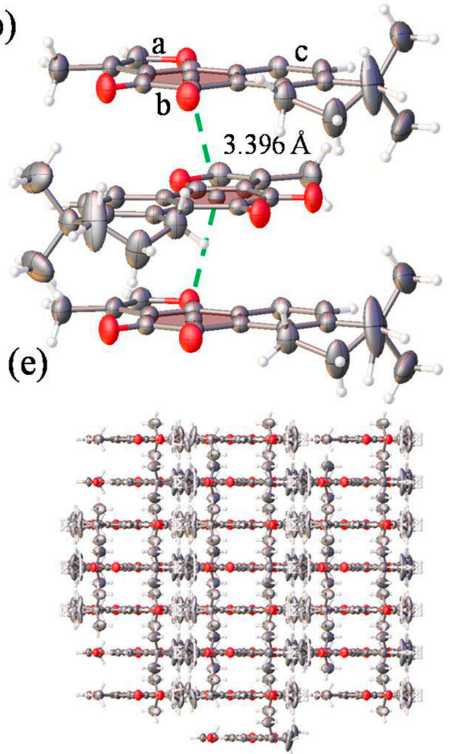

Figure 1. (a) The molecular structure of Tan-IIA; (b) Stacking interactions in a step showing strong overlap for Tan-IIA. The step showed two benzene rings with a diketone group stacking interactions occurring with distances to the ring centroid of $3.396 \AA$. The pores viewed along the a axis (c), b axis (d) and c axis (e). 


\subsection{The Lethal Effects of Tan-IIA on Embryos}

The lethal effects of Tan-IIA on zebrafish embryos were recorded at $24 \mathrm{hpf}, 48 \mathrm{hpf}, 72 \mathrm{hpf}$ and $96 \mathrm{hpf}$, as shown in Figure 2, and at $12 \mathrm{hpf}$ as shown in Tables S2 and S3. In the chorionic embryo group, compared with the control group, little obvious lethal effect at concentrations of $1 \mu \mathrm{M}$ and $3 \mu \mathrm{M}$ was observed, but with the increasing concentration of Tan-IIA treatment, severe abnormalities of heart and pericardium occurred, which were observed in a dose-dependent manner. The mortality at $24 \mu \mathrm{M}$ for $96 \mathrm{hpf}$ was just 51.6\% (Figure 2a). Meanwhile in the dechorionated embryo group, lethal effect appeared at a concentration of $5 \mu \mathrm{M}$, even reaching $100 \%$ mortality $(50 \mu \mathrm{M}$ at $72 \mathrm{hpf}$ and $20 \mu \mathrm{M}$ at $96 \mathrm{hpf}$, as shown in Figure 2b). The $\mathrm{LC}_{50}$ values in the dechorionated embryo group at $72 \mathrm{hpf}$ and $96 \mathrm{hpf}$ were $18.5 \mu \mathrm{M}$ and $12.8 \mu \mathrm{M}$, respectively. The hatchability of the chorionic embryo group is shown in Figure $2 \mathrm{c}$, which indicated that high concentrations of Tan-IIA $(12 \mu \mathrm{M}, 24 \mu \mathrm{M})$ affected the hatchability of the embryos. In the dechorionated embryo group, the hatchability could not be calculated due to the chorion being removed. The main lethal endpoints observed in the dechorionated embryo group were coagulated embryos at $48 \mathrm{hpf}$ and lack of heartbeat at $96 \mathrm{hpf}$; lack of somite formation and non-detachment of the tail were also observed in some cases. The abovementioned results indicated that Tan-IIA exhibited some toxicity to zebrafish embryos in a dose-dependent manner.

(a)

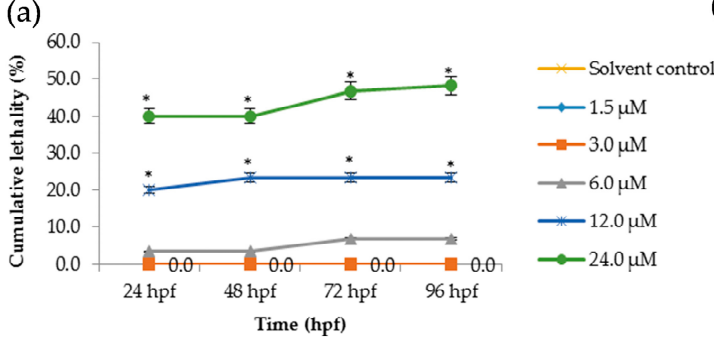

(b)

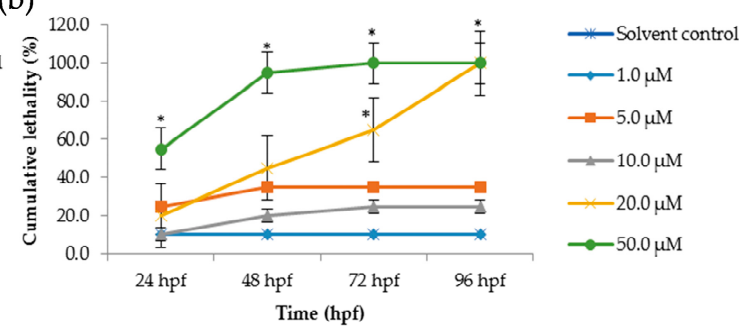

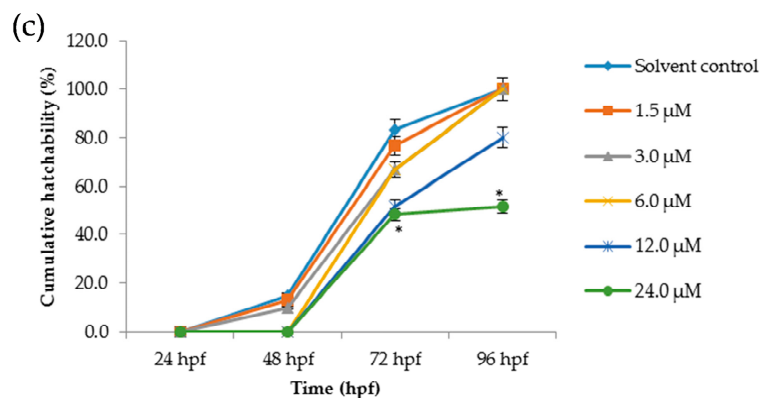

Figure 2. Cumulative lethality and hatchability curves of embryos exposed to different concentrations of Tan-IIA. (a) Cumulative lethality curves of chorionic embryos; (b) Cumulative lethality curves of dechorionated embryos; (c) Cumulative hatchability curves of chorionic embryos. $(n=20$ zebrafish per treatment; ${ }^{*} p<0.05$, compared to control).

\subsection{The Teratogenic Effects of Tan-IIA on Embryos}

The morphological changes of zebrafish embryos induced by Tan-IIA were further evaluated. Zebrafish have rapidly developed into a promising model for whole-organism toxicology screening. The influence of Tan-IIA on the development of Zebrafish embryos was determined, as shown in Figure 3 and Figure S1 (12 hpf), and the detailed data are listed in Table S4.

As shown in Figure 3a, in the chorionic embryo group, the zebrafish embryos without Tan-IIA treatment had developed normally, but following treatment with an increasing dosage of Tan-IIA, the hatched fish developed obvious pericardial edema at $6 \mu \mathrm{M}$ for $96 \mathrm{hpf}$ and spinal curvature at $24 \mu \mathrm{M}$ for $96 \mathrm{hpf}$. Moreover, after treatment with Tan-IIA $\geq 6 \mu \mathrm{M}$, the time it took for the embryos to mature into fish was longer than that for the control group, which suggested that Tan-IIA exhibited growth inhibition of zebrafish embryos. Furthermore, in the dechorionated embryo group, the zebrafish embryos without 
chorions could uptake larger amounts of drugs. It was found that the embryos all died at $20 \mu \mathrm{M}$ for $96 \mathrm{hpf}$ and $50 \mu \mathrm{M}$ for $72 \mathrm{hpf}$, and the growth of embryos were inhibited seriously at $50 \mu \mathrm{M}$. In a word, above results suggested that Tan-IIA, in a concentration-dependent and low time-dependent manner, exhibited certain toxicity and growth inhibition in zebrafish embryos in vivo at high concentrations.

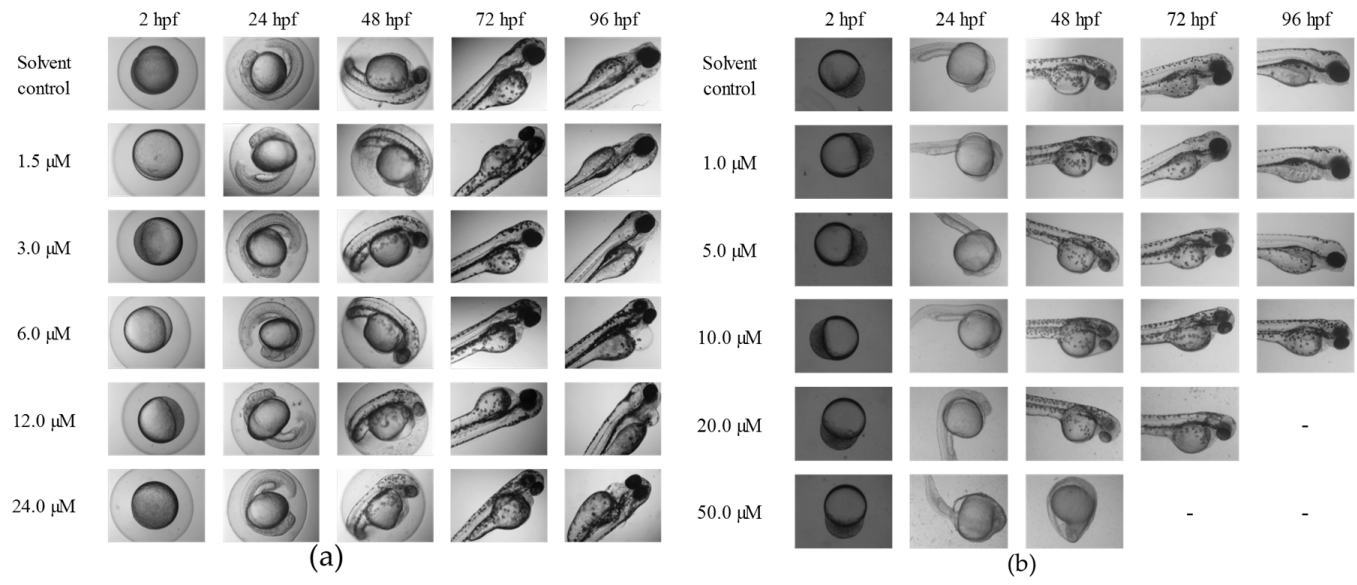

Figure 3. Morphology of zebrafish embryos exposed to Tan-IIA. (a) Morphology of chorionic embryos; (b) Morphology of dechorionated embryos-denotes that the treated embryos were all dead.

Moreover, with the protection of the chorion, the zebrafish hatched from eggs exposed to different concentrations $(0,1.5$ and $3 \mu \mathrm{M})$ of Tan-IIA for $96 \mathrm{hpf}$ displayed great health conditions, but the zebrafish treated with $6 \mu \mathrm{M}$ showed abnormal development with pericardial edema and scoliosis, as well as some with the tail missing at $24 \mu \mathrm{M}$. However, without the protection of the chorion, the zebrafish were subjected to mass mortality and mild teratogenic effects at $5 \mu \mathrm{M}$ for $96 \mathrm{hpf}$. All of the major malformations of scoliosis, tail autolysis, and pericardial edema were observed at the highest concentration of $50 \mu \mathrm{M}$ (Figure 4b). Especially in groups treated with $20 \mu \mathrm{M}$ and $50 \mu \mathrm{M}$ of Tan-IIA, the zebrafish pericardium was enlarged, edematous and congested, which appeared to be about two-times larger than that of the control group. The longitudinal sections of pericardial edema and scoliosis with hematoxylin-eosin (HE) staining were shown in Figure S2. These results indicated that Tan-IIA exhibited potential cardiotoxicity and growth inhibition in zebrafish embryos.

(a)

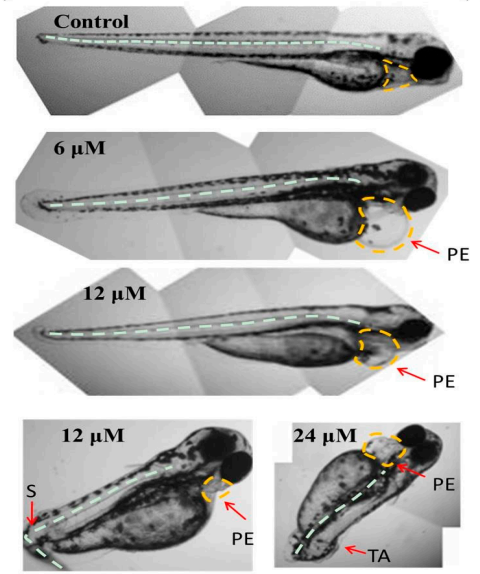

(b)

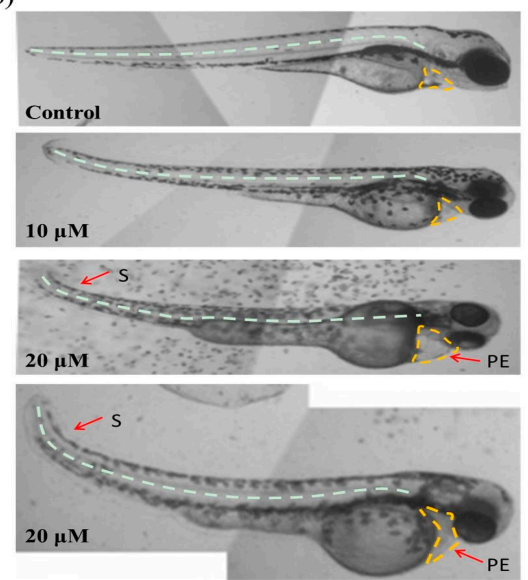

Figure 4. Abnormal embryos exposed to Tan-IIA. (a) Abnormal embryos in the chorionic embryo group; (b) Abnormal embryos in the dechorionated embryo group. S: scoliosis; PE: pericardial edema; TA: tail autolysis. 


\section{Discussion}

Although Salvia miltiorrhiza has been applied for many years, the toxicity of the mono-constituent of Salvia miltiorrhiza, tanshinone IIA, has not been fully studied. Cao Xiaomei et al. [23] used cavy and rabbit to evaluate the hemolytic effect, hormesis effect and anaphylaxis of sodium tanshinone IIA sulfonate injection. Our research explored the developmental toxicity and acute toxicity of Tan-IIA by using chorionic and dechorionated zebrafish embryos. The lethality and teratogenicity were observed and summarized at different concentrations of Tan-IIA.

The chorion of a zebrafish embryo is the acellular envelope surrounding embryo. The role of the chorion is to protect the embryo from damage and to prevent polyspermy [24,25]. As shown in Figure $2 \mathrm{a}, \mathrm{b}$, the lethality of the chorionic embryo group was lower than dechorionated embryo group at similar concentrations of Tan-IIA. In the dechorionated embryo group, the $\mathrm{LC}_{50}$ values at $72 \mathrm{hpf}$ and $96 \mathrm{hpf}$ were $18.5 \mu \mathrm{M}$ and $12.8 \mu \mathrm{M}$, respectively, while in the chorionic embryo group, the lethality of the highest concentration $(24 \mu \mathrm{M})$ was below $50 \%$. These results indicated that Tan-IIA may be partly blocked by the chorion. The incubation of the zebrafish embryo is the result of hatching enzymes and mechanical force [26]. In the process of hatching, the chorion is decomposed by hatching enzymes and then the embryo, with warp and sway movements, would break through the chorion $[27,28]$. As shown in Figure $2 b$, the hatchability of the embryos declined at a concentration of $12 \mu \mathrm{M}$ and $24 \mu \mathrm{M}$, which revealed that Tan-IIA in high concentrations might affect the functions of hatching enzymes and/or the motor system of the embryo.

The development of zebrafish is very similar to mammals in most aspects of embryo development, including early embryonic processes and the development of cardiovascular, somite and skeletal structures, etc. [11,12]. When the embryos were exposed to chemicals upon fertilization, teratogenic effects could be observed in the following several days. As shown in Figure 4 and Table S4, there were no teratogenic effects when the concentration of Tan-IIA was below $5 \mu \mathrm{M}$ in both the chorionic and dechorionated embryo groups. The major malformations observed were scoliosis, tail autolysis, and pericardial edema. Due to the resistance function of the chorion, the appearance of teratogenic effect was delayed compared to the dechorionated embryo group.

\section{Materials and Methods}

\subsection{Materials and Reagents}

Tanshinone IIA (Tan-IIA) was purchased from Damao Chemical Reagent Factory (Tianjin, China), protease E from Sigma-Aldrich (Guangzhou, China), dimethyl sulfoxide (DMSO) from Guangzhou Chemical Reagent Factory (Guangzhou, China), the 6-well plate from Guangzhou JET Bio-Filtration Co., Ltd. (Guangzhou, China), and the inverted optical microscope from Chongqing Optec Instrument Co., Ltd. (Chongqing, China).

\subsection{Brood Zebrafish Maintenance and Egg Production}

Zebrafish were maintained according to The Zebrafish Book [29]. Wild type Tuebingen strain zebrafish (Danio rerio) were obtained from China Zebrafish Resource Center, CZRC (Wuhan, China), and three-month-old zebrafish were used for egg production. Sexually mature zebrafish were maintained in a recirculating aquaculture system (Shanghai Haisheng Biotech Co., Ltd, Shanghai, China) at $28.0 \pm 1.0^{\circ} \mathrm{C}$. The water was purified by water purifier (Shenzhen Luoke Water Purification Equipment Co., Ltd, Shenzhen, China), and $\mathrm{NaHCO}_{3}$ and $\mathrm{NaCl}$ were used to adjust the $\mathrm{pH}$ and conductivity at pH 6.8 7.4 and 500 550 $\mu$ S, respectively. The housing system was equipped with a temperature control unit, UV light, and activated carbon filter system. The day to night photoperiod was $14 \mathrm{~h}$ : $10 \mathrm{~h}$. Mature zebrafish were fed with brine shrimp twice and powder feed once daily.

Males and females at a ratio of 2:1 or 1:1 were placed in spawning tanks a few hours before the onset of darkness on the day prior to the test. For the collection of eggs, spawn traps were placed into the spawning tanks, and the spawn traps were covered with inert wire mesh of appropriate 
mesh size. About 30 min after the onset of light, spawn traps were removed and eggs were collected. To avoid genetic bias, eggs were collected from a minimum of three breeding groups, mixed and randomly selected.

\subsection{The Developmental Toxicity Assay}

The developmental toxicity assay was carried out according to the fish embryo acute toxicity (FET) test, developed from Organization for Economic Cooperation and Development (OECD) guidance [30] and the book entitled Zebrafish: Methods for Assessing Drug Safety and Toxicity with some modifications [31,32].

\subsubsection{Removal of Chorion}

The method of removing the chorion was taken from the published protocol [33]. Eggs were placed in a $90 \mathrm{~mm}$ petri dish and the water was sopped up. $5 \mathrm{~mL}$ of pronase $(0.208 \mathrm{~g} / \mathrm{L}$, warmed to $28.5^{\circ} \mathrm{C}$ ) was poured into the petri dish and incubated for $6 \mathrm{~min}$. $20 \mathrm{~mL}$ fish water was added to the petri dish, and the eggs were allowed to sink to the bottom of the petri dish before the water was slowly poured out. The eggs were gently rinsed three times with fish water and most of the chorions had thus been removed.

\subsubsection{Embryo Exposure}

In the preliminary experiment, the concentrations of Tan-IIA of $1.5 \mu \mathrm{M}, 3.0 \mu \mathrm{M}, 6.0 \mu \mathrm{M}, 12.0 \mu \mathrm{M}$, $24.0 \mu \mathrm{M}$ were selected. Tan-IIA was dissolved in DMSO with a final solvent concentration of $0.2 \%$ in the test solution. $5 \mathrm{~mL}$ of these solutions and 20 chorionic embryos of $2 \mathrm{~h}$ post-fertilization (hpf) were transferred to 6-well plates. Each test concentration and solvent control were on the same plate, amounting to three parallel experimental plates and one negative control plate of fish water. The embryos were incubated at $28.0 \pm 1.0^{\circ} \mathrm{C}$ with the photoperiod of $14 \mathrm{~h}: 10 \mathrm{~h}$.

According to the results of the pre-test, the concentrations of Tan-IIA were changed to $1.0 \mu \mathrm{M}$, $5.0 \mu \mathrm{M}, 10.0 \mu \mathrm{M}, 20.0 \mu \mathrm{M}$, and $50.0 \mu \mathrm{M}$, with the final DMSO concentration of $0.4 \%$ in the test solution. In addition, normal dechorionated embryos of $2 \mathrm{~h}$ post-fertilization (hpf) were used for exposure. The other methods were the same as the preliminary experiment described above.

\subsubsection{Observation of Mortal and Teratogenic Effects}

Observations were recorded using an inverted optical microscope every $24 \mathrm{~h}$ until the end of the test, including coagulation of embryos, lack of somite formation, non-detachment of the tail, and lack of heartbeat. Any positive outcomes in these observations indicated that the zebrafish embryo was dead. The teratogenic embryos were photographed to analyze the deformed parts. $\mathrm{LC}_{50}$ were calculated at $72 \mathrm{hpf}$ and $96 \mathrm{hpf}$.

\subsection{Statistical Analysis}

The significance of mortality and hatching rates were determined by chi-square $\left(\chi^{2}\right)$ test and Fisher's exact test, because both death and hatching are categorical variables. Significant differences were considered at $p<0.05$. Statistical analysis was conducted using SPSS 17.0 (SPSS Statistics 17.0.1, International Business Machines Corp., Armonk, NY, USA).

\section{Conclusions}

Tanshinone IIA, the main active ingredient of Salvia miltiorrhiza, is an important drug to treat diseases of cardiovascular system such as hypertension and atherosclerosis. The in vitro potential toxicity of Tan-IIA was evaluated by the zebrafish embryo model. It was found that Tan-IIA exhibited severe growth inhibition, development malformation and cardiotoxicity at high concentrations. Sodium tanshinone IIA sulfonate injection has clinical applications at a dose of 40-80 mg per day, which 
is a low concentration compared to those investigated in this study applied to zebrafish. Therefore, sodium tanshinone IIA sulfonate injection may cause minimal side effects at a clinical dose, which is consistent with the results of clinical research [34]. This study firstly reported the potential toxicity of Tan-IIA at high concentrations on zebrafish, pointing out the potential risk of its clinical application at an increased dose.

Supplementary Materials: Supplementary materials are available online, including Figures S1 and S2.

Acknowledgments: The authors acknowledge the National Nature Science Foundation of China (81572926), the Provincial Major Scientific Research Projects in Universities of Guangdong Province (2014KZDXM053), the Joint Natural Sciences Fund of the Department of Science and Technology and the First Affiliated Hospital of Guangdong Pharmaceutical University (GYFYLH201309), and Traditional Chinese Medicine Bureau of Guangdong Province (20151265).

Author Contributions: Tao Wang and Kangdi Zheng performed the experiments; Wenjie Mei and Chengxi Wang conceived and designed the experiments; Qiong Wu and Kangdi Zheng contribute to the single crystal growth and data analysis, Jiaojiao Chen and Yao Qin analyzed the data; Yutao Lan contributed to materials and analysis tools; Tao Wang and Qiong Wu wrote the paper; Baoguo Wang and Wenjie Mei revised the paper. All the authors read and approved the final manuscript.

Conflicts of Interest: The authors declare no conflict of interest.

\section{References}

1. Gao, S.; Liu, Z.P.; Li, H.; Little, P.J.; Liu, P.Q.; Xu, S.W. Cardiovascular actions and therapeutic potential of tanshinone IIA. Atherosclerosis 2012, 220, 3-10. [CrossRef] [PubMed]

2. Xu, S.W.; Liu, P.Q. Tanshinone II-A: New perspectives for old remedies. Expert. Opin. Ther. Pat. 2013, 23, 149-153. [CrossRef] [PubMed]

3. Zhou, L.M.; Zuo, Z.; Chow, M.S.S. Danshen: An overview of its chemistry, pharmacology, pharmacokinetics, and clinical use. J. Clin. Pharmacol. 2005, 45, 1345-1359. [CrossRef] [PubMed]

4. Li, W.; Zhang, Y.; Xing, C.Y.; Zhang, M.Y. Tanshinone IIA represses inflammatory response and reduces radiculopathic pain by inhibiting IRAK-1 and NF-kB/p38/JNK signaling. Int. Immunopharmacol. 2015, 28, 382-389. [CrossRef] [PubMed]

5. Chen, W.Y.; Tang, F.T.; Xie, B.L.; Chen, S.R.; Huang, H.Q.; Liu, P.Q. Amelioration of atherosclerosis by tanshinone IIA in hyperlipidemic rabbits through attenuation of oxidative stress. Eur. J. Pharmacol. 2012, 674, 359-364. [CrossRef] [PubMed]

6. Shu, M.; Hu, X.R.; Hung, Z.A.; Huang, D.D.; Zhang, S. Effects of tanshinone IIA on fibrosis in a rat model of cirrhosis through heme oxygenase-1, inflammation, oxidative stress and apoptosis. Mol. Med. Rep. 2016, 13, 3036-3042. [PubMed]

7. Wang, P.; Zhou, S.G.; Xu, L.P.; Lu, Y.; Yuan, X.; Zhang, H.J.; Li, R.F.; Fang, J.; Liu, P.Q. Hydrogen peroxide-mediated oxidative stress and collagen synthesis in cardiac fibroblasts: Blockade by tanshinone IIA. J. Ethnopharmacol. 2013, 145, 152-161. [CrossRef] [PubMed]

8. Zhang, Y.; Jiang, P.X.; Ye, M.; Kim, S.H.; Jiang, C.; Lü, J.X. Tanshinones: Sources, pharmacokinetics and anti-cancer activities. Int. J. Mol. Sci. 2012, 13, 13621-13666. [CrossRef] [PubMed]

9. McGrath, P.; Li, C.Q. Zebrafish: A predictive model for assessing drug-induced toxicity. Drug. Discov. Today 2008, 13, 394-401. [CrossRef] [PubMed]

10. Hill, A.J.; Teraoka, H.; Heideman, W.; Peterson, R.E. Zebrafish as a model vertebrate for investigating chemical toxicity. Toxicol. Sci. 2005, 86, 6-19. [CrossRef] [PubMed]

11. Yang, L.X.; Ho, N.Y.; Alshut, R.; Legradi, J.; Weiss, C.; Reischl, M.; Mikut, R.; Liebei, U.; Muller, F.; Strahle, U. Zebrafish embryos as models for embryotoxic and teratological effects of chemicals. Reprod. Toxicol. 2009, 28, 245-253. [CrossRef] [PubMed]

12. McCollum, C.W.; Ducharme, N.A.; Bondesson, M.; Gustafsson, J.A. Developmental toxicity screening in zebrafish. Birth. Defects Res. C. Embryo. Today 2011, 93, 67-114. [CrossRef] [PubMed]

13. McClain, R.M.; Keller, D.; Casciao, D.; Fu, P.; MacDonald, J.; Popp, J.; Sagarta, J. Neonatal mouse model: Review of methods and results. Toxicol. Pathol. 2001, 29, 128-137. [CrossRef] [PubMed]

14. Foote, R.H.; Carney, E.W. The rabbit as a model for reproductive and developmental toxicity studies. Reprod. Toxicol. 2000, 14, 477-493. [CrossRef] 
15. Li, Q.; Wang, P.P.; Chen, L.; Gao, H.W.; Wu, L.L. Acute toxicity and histopathological effects of naproxen in zebrafish (Danio rerio) early life stages. Environ. Sci. Pollut. Res. Int. 2016, 23, 18832-18841. [CrossRef] [PubMed]

16. Lin, T.; Zhou, D.J.; Dong, J.; Jiang, F.C.; Chen, W. Acute toxicity of dichloroacetonitrile (DCAN), a typical nitrogenous disinfection by-product (N-DBP), on zebrafish (Danio rerio). Ecotoxicol. Environ. Saf. 2016, 133, 97-104. [CrossRef] [PubMed]

17. Bugel, S.M.; Bonventre, J.A.; Tanguay, R.L. Comparative developmental toxicity of flavonoids using an integrative zebrafish system. Toxicol. Sci. 2016, 154, 55-68. [CrossRef] [PubMed]

18. Fong, H.C.; Ho, J.C.; Cheung, A.H.; Lai, K.P.; William, K.F. Developmental toxicity of the common UV filter, benophenone-2, in zebrafish embryos. Chemosphere 2016, 164, 413-420. [CrossRef] [PubMed]

19. Liu, L.H.; Li, Y.F.; Coelhan, M.; Chan, H.M.; Ma, W.L.; Liu, L.Y. Relative developmental toxicity of short-chain chlorinated paraffins in Zebrafish (Danio rerio) embryos. Environ. Pollut. 2016, 219, 1122-1130. [CrossRef] [PubMed]

20. Xu, C.; Tu, W.Q.; Deng, M.; Jin, Y.X.; Lu, B.; Zhang, C.N.; Lin, C.M.; Wu, Y.M.; Liu, W.P. Stereoselective induction of developmental toxicity and immunotoxicity by acetochlor in the early life stage of zebrafish. Chemosphere 2016, 164, 618-626. [CrossRef] [PubMed]

21. Zheng, K.D.; Chen, Z.S.; Wang, C.X.; Zeng, R.F.; Wang, M.C.; Mei, W.J.; Zhang, W.D. Toxic effect of dimethyl sulfoxide on zebrafish embryo. J. Guangdong. Pharm. Univ. 2014, 30, 636-650.

22. Xiong, X.Q.; Luo, S.; Wu, B.L.; Wang, J.W. Comparative developmental toxicity and stress protein responses of dimethyl sulfoxide to rare minnow and zebrafish embryos/larvae. Zebrafish 2017, 14, 60-68. [CrossRef] [PubMed]

23. Cao, X.M.; Chen, X.M.; Sun, W.L. Safety of sodium tanshinone IIA sulfonate injection. J. Med. Postgrad. 2010, 23, 474-476.

24. Kim, D.H.; Hwang, C.N.; Sun, Y.; Lee, S.H.; Kim, B.; Neson, B.J. Mechanical analysis of chorion softening in prehatching stages of zebrafish embryos. IEEE. Trans. Nanobioscience 2006, 5, 89-94. [CrossRef] [PubMed]

25. Kim, D.H.; Sun, S.; Kim, B.; Hwang, C.N.; Lee, S.H.; Nelson, B.J. Mechanical property characterization of the zebrafish embryo chorion. Conf. Proc. IEEE. Eng. Med. Biol. Soc. 2004, 7, 5061-5064. [PubMed]

26. Ong, K.J.; Zhao, X.X.; Thistle, M.E.; MacCormack, T.J.; Clark, R.J.; Ma, G.B.; Martinez-Rubi, Y.; Simard, B.; Loo, J.S.C.; Veinot, J.G.; et al. Mechanistic insights into the effect of nanoparticles on zebrafish hatch. Nanotoxicology 2014, 8, 295-304. [CrossRef] [PubMed]

27. Okada, A.; Sano, K.; Nagata, K.; Yasumasu, S.; Ohtsuka, J.; Yamamura, A.; Kubota, K.; Iuchi, I.; Tanokura, M. Crystal structure of zebrafish hatching enzyme 1 from the zebrafish Danio rerio. J. Mol. Biol. 2010, 402, 865-878. [CrossRef] [PubMed]

28. Hiroi, J.; Maruyama, K.; Kawazu, K.; Kaneko, T.; Ohtani-Kaneko, R.; Yasumasu, S. Structure and developmental expression of hatching enzyme genes of the Japanese eel Anguilla japonica: An aspect of the evolution of fish hatching enzyme gene. Dev. Genes. Evol. 2004, 214, 176-184. [CrossRef] [PubMed]

29. Westerfield, M. The Zebrafish Book, 4th ed.; University of Oregon Press: Eugene, OR, USA, 2000; pp. 1-196.

30. OECD. Test No. 236: Fish Embryo Acute Toxicity (FET) Test; OECD Publishing: Paris, France, 2013.

31. Haldi, M.; Harden, M.; D'Amico, L.; DeLise, A.; Seng, W.L. Developmental toxicity assessment in zebrafish. In Zebrafish: Methods for Assessing Drug Safety and Toxicity, 1st ed.; McGrath, P., Ed.; John Wiley \& Sons, Inc.: Hoboken, NJ, USA, 2012; pp. 15-25.

32. McGrath, P. Use of emerging models for developmental toxicity testing. In Zebrafish: Methods for Assessing Drug Safety and Toxicity, 1st ed.; McGrath, P., Ed.; John Wiley \& Sons, Inc.: Hoboken, NJ, USA, 2012; pp. $27-44$.

33. Thisse, C.; Thisse, B. High-resolution in situ hybridization to whole-mount zebrafish embryos. Nat. Protoc. 2008, 3, 59-69. [CrossRef] [PubMed]

34. Ma, X.C.; Li, J.X.; Xu, M.D.; Deng, X.Q.; Yue, G.H.; Zheng, J.H. Meta-analysis on effectiveness and safety of tanshinone IIa sulfonate sodium injection in treatment of unstable angina pectoris. World Sci. Technol. Mod. Tradit. Chin. Med. 2015, 17, 1766-1774.

Sample Availability: Samples of the compounds are available from the authors.

(C) 2017 by the authors. Licensee MDPI, Basel, Switzerland. This article is an open access article distributed under the terms and conditions of the Creative Commons Attribution (CC BY) license (http:/ / creativecommons.org/licenses/by/4.0/). 